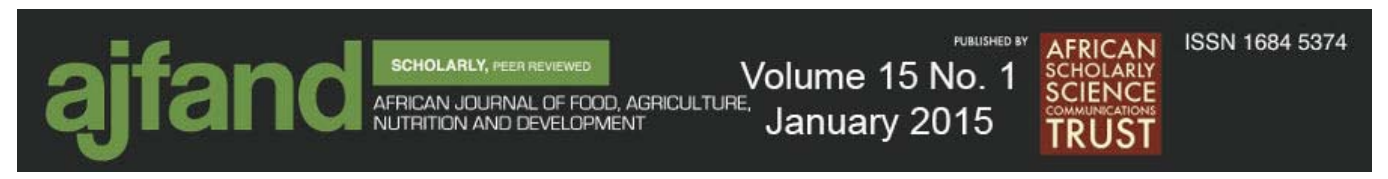

\title{
BOVINE IN-VITRO EMBRYO PRODUCTION AND ITS CONTRIBUTION TOWARDS IMPROVED FOOD SECURITY IN KENYA
}

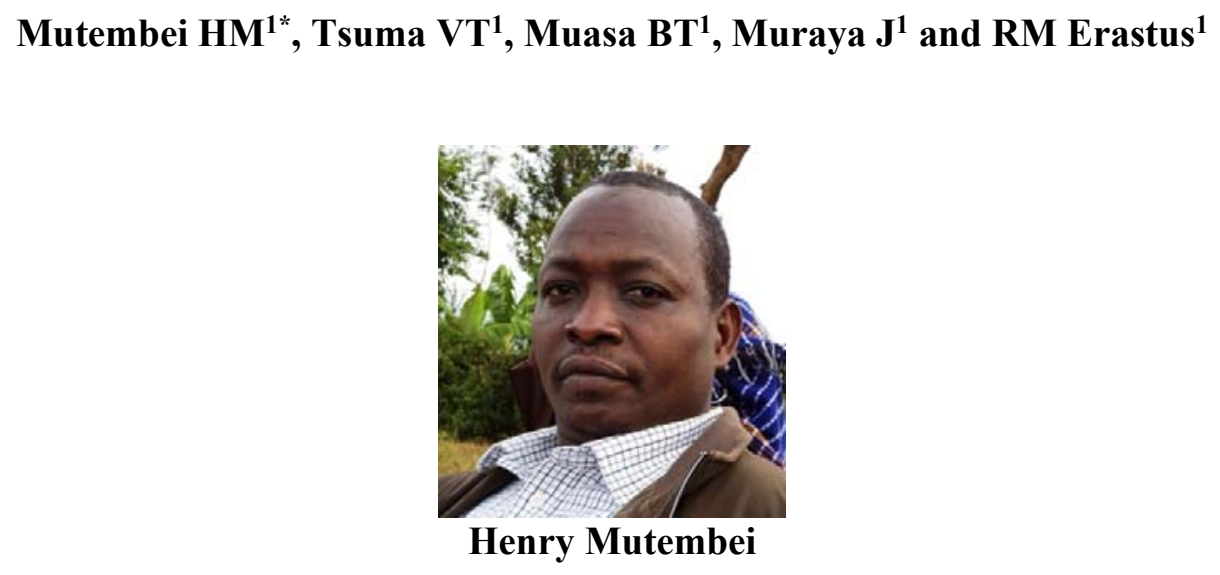

*Corresponding author email: hmutembei@uonbi.ac.ke

${ }^{1}$ Clinical Studies Department, University of Nairobi, P.O. Box 29053-0025, Nairobi, Kenya. 


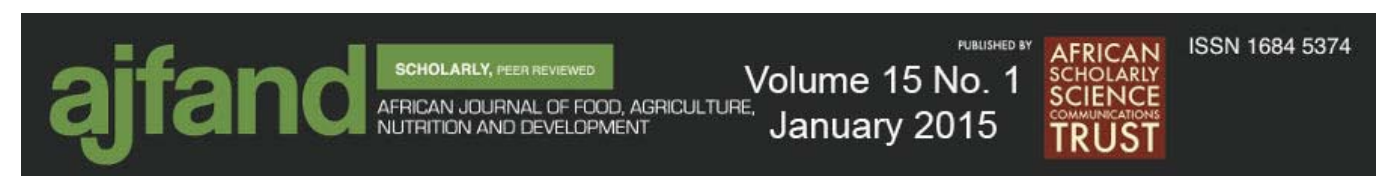

\section{ABSTRACT}

The Boran breed is mainly kept by pastoralist communities as a source of both milk and beef, and by commercial beef ranches mainly for beef production. Although this breed Boran may seemingly be low valued, it can be raised to higher reproductive potential using current reproductive technologies such as in-vitro embryo production and embryo transfer. In this author's literature search there was lack of locally optimized procedures for boosting the reproductive potential of the Boran cow using such technologies. This paper discusses simplified methods used for in-vitro embryo production and embryo transfer that have been tested and optimized for the Kenyan Boran cows as described in text. This article provides the reader with technical procedures, the outcomes and the challenges experienced during use of the technologies. The results of the reported in vitro embryo production obtained an oocyte maturation rate of over $90 \%$, over $70 \%$ cleavage rate and an embryo output of 30-50\% blastocyst rate. Embryo transfer had a conception rate of $45-50 \%$. All the 17 calves born out of this work have since attained puberty without any abnormalities. This research was the first to produce a "test tube" calf in Kenya and built the capacity of 57 staff on these technical procedures within East Africa. Utilization of the in vitro embryo production technology in Kenya can be used to do value addition to indigenous cows and improve household incomes and food security. This avenue can be used to raise household revenues and attract many more farmers to engage in farming leading to improved national economic and food security. Kenya can utilize reproductive technologies in the dairy sector due to the massive potential benefits to revolutionize the sector for enhanced food security by increasing cattle productivity. The paper highlights practical ways of applying the technologies discussed therein to help raise the economy of the Kenyan livestock farmers and boost their food security.

Key words: Reproductive Technologies, Boran cattle, Kenya 


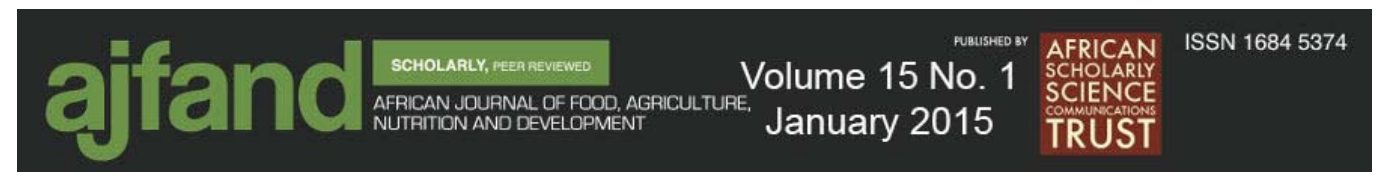

\section{INTRODUCTION}

The world food crisis and rise in demand for food of animal origin, competitive global markets for such foods and the negative effects of climatic change, especially in the marginal tropical areas [1], demand that innovative strategies and interventions be urgently employed [2]. The dairy sector in developing countries, especially in low input production systems, has the potential of providing cost effective animal protein requirements. To do this, such units need to be efficient, and the starting point is using appropriate genotypes. Many attempts aimed at improving the productivity of indigenous dairy cattle in developing countries through crossbreeding with temperate breeds have generally not been successful [3].

Many studies have shown that temperate dairy breeds best combine with local tropical breeds at $50 \%$ of each breed composition ( $\mathrm{F}_{1}$ cross). The $\mathrm{F}_{1}$ is well adapted and produces reasonable amount of milk under the local conditions [3, 4].

Aggressive promotion of a few exotic cattle breeds in sub-Saharan Africa (SSA) has resulted in cattle genotypes that are not only poorly adapted to the production systems but are also commercially inefficient [5].

In order to meet the increasing demand for appropriate improved dairy cattle genotypes in the marginal dairy systems in the Eastern African region, more efficient production and delivery of improved germplasm is needed. Breeding strategies that utilize a combination of emerging reproductive technologies, especially the in-vitro embryo production (IVEP), ovum pick up (OPU) and semen sexing, offer prudent possible options [6]. For example, indigenous breeds such as the Boran that are inferior commercial milk producers would be valuable as oocyte donors as well as surrogates for crossbred embryos under IVEP-based systems.

Up to $80 \%$ of Kenya's landmass is arid or semi-arid (ASAL). The pastoralists who live in the ASAL depend heavily on livestock as their most viable source of livelihood. Among the Zebu breeds of livestock kept in these areas, the most commercially viable breed in Kenya is the Boran.

Conventional embryo transfer technology is based on the superovulation of a highquality donor animal and the subsequent recovery of embryos by flushing the uterus a week after breeding to the bull of choice $[7,8,9]$. In cattle, this technology is well established. The technology can be advanced following further understanding of the reproductive physiology of the cow by the practitioners of the Embryo Transfer (ET) in Kenya so as to meet the demand for better bovine genetic improvement within Eastern African countries.

An alternative to avoid the more expensive conventional superovulation procedures during ET is in-vitro embryo production (IVEP) [10]. This technology allows for cheaper production of a predictable supply of embryos from ovaries of live or slaughtered cows. From live selected animals, repeated recovery of primary oocytes using ovum pick up procedures (OPU) is possible [7,8]. To date in Kenya, our team 


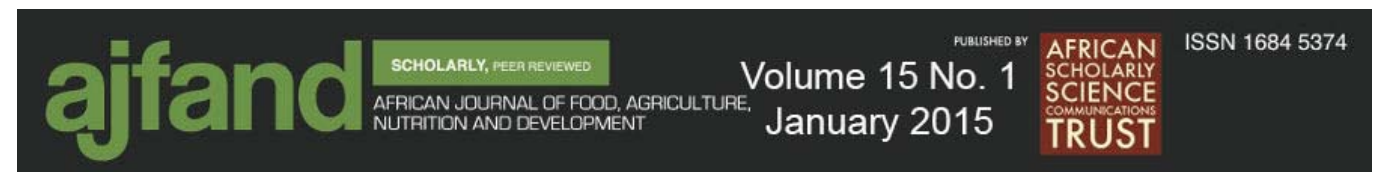

has shown that IVEP and OPU technique have given considerable success in cattle, achieving a success rate ranging from $30-50 \%$ for development of pre-implantation stage embryo in-vitro and conception rates of up to $50 \%$. The IVEP technology does not only offer optimization of high-quality dams (mothers), but also allows the preservation and rapid multiplication of genetically superior cows by making embryos available for sexing, cloning and nuclear transfer [11]. Use of such advances in technologies can be utilized to turn around the economic gains of farmers.

Utilization of the IVEP technology in Kenya can be used t undertake value addition to indigenous cows and improve household incomes and food security. Farmers keeping low-grade cows can enter into commercial contracts to provide these cows as surrogate recipients for production of heifer calves. In the process, owners of such cows will have a valued product to sell for income and be left with milk for use by family members. This avenue can be used to raise household revenues and attract many more farmers to engage in farming, leading to improved national economic and food security. Kenya can utilize reproductive technologies in the dairy sector due to their massive potential benefits to revolutionize the sector for enhanced food security by increasing cattle productivity.

This paper highlights the practical ways of applying IVEP technologies to help turn around the economy of the Kenyan livestock farmers and boost food security. It discusses the technical aspects of the procedures involved in the in-vitro production of bovine embryos and embryo transfer, with special reference to the application of the techniques in Kenya.

\section{MATERIALS AND METHODS}

Production of embryos in the laboratory involved four major steps: 1) Ovary collection, 2; Oocyte maturation, 3) in vitro fertilization and 4) Embryo culture.

In brief, ova were collected from live Boran cows in Kapiti Ranch using ovum pick-up (OPU) technology and transported to the laboratory in a warm saline solution (supplemented with $0.1 \mathrm{~g} / \mathrm{L}$ streptomycin). In some cases, ovaries from slaughtered animals were used. Pre-slaughter body condition scores (BCS) were assessed prior to slaughter at the holding area. Ova collected from cows were delivered to the laboratory within 4 hrs of collection.

Cumulus oocyte complexes (COCs) were obtained by aspiration of 3 to $8 \mathrm{~mm}$ follicles using a 21-gauge needle attached to a $10 \mathrm{~mL}$ syringe and manipulated in Tyrodes Albumin Pyruvate (TALP) Hepes medium supplemented with $0.4 \%$ bovine serum albumin. The COCs were graded morphologically according to oocyte cytoplasm aspect and morphology of overlying zona pelucida cumulus cell layers; only grade A and B COCs were used for the work. These were COCs with compact cumulus cell layers and oocytes with homogenous (grade A) or slightly heterogeneous (grade B) cytoplasm. Standard in vtro oocyte maturation, fertilization and embryo culture procedures were conducted. Synchronization of the recipient cows was achieved by 


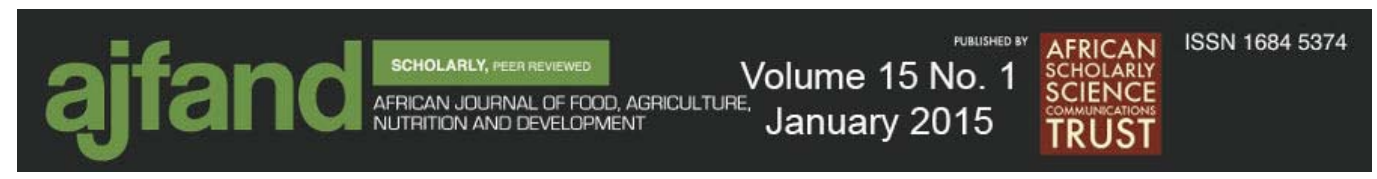

knocking off the corpous luteum using cloprostenol 500ug ( $2 \mathrm{ml}$ of lutealyase) and embryo transfer was done at day seven using in vitro produced blastocysts.

All chemicals used were from Sigma Chemical Co. (St. Louis, MO, USA), unless otherwise stated.

\section{RESULTS}

\section{Outputs of IVEP}

From this IVEP work, data collected on the donor cows and results obtained in the laboratory at various levels are shown in Table 1-2.

The BCS of the sampled animals ranged from 1 to 5 . Seventy four (74) $\%$ of them had BCS of $1.5-2.5$ (Figure 1).

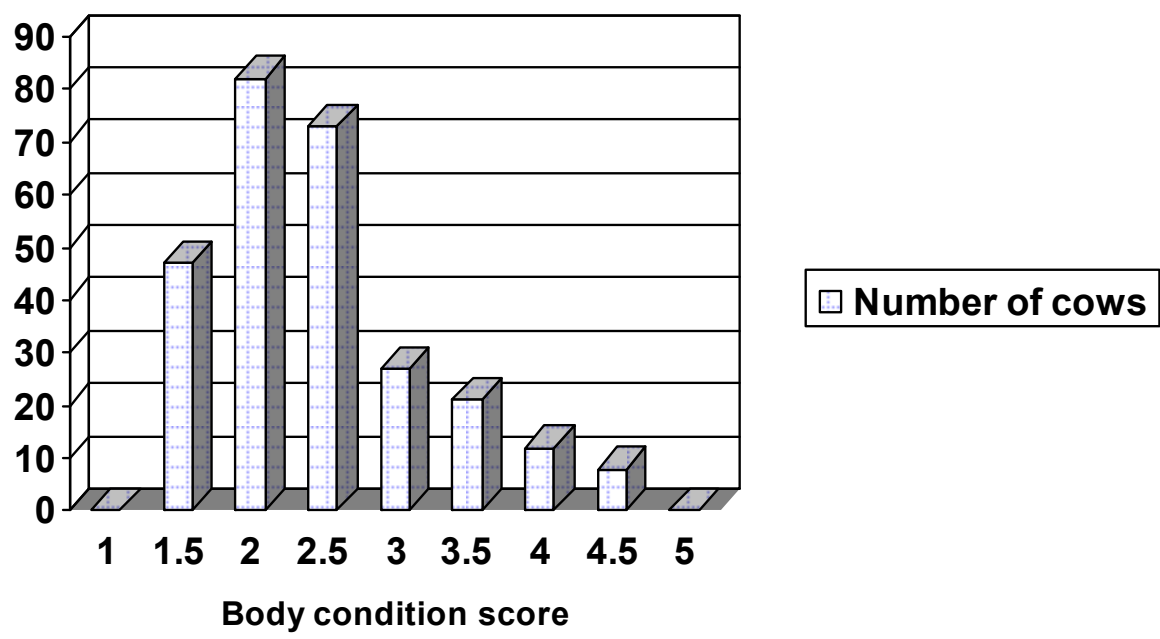

\section{Figure 1: Distribution of pre-slaughter body condition scores of the sampled animals}

Forty two (42) \% of the cows sampled at slaughter were pregnant at different stages of gestation. Overall, 163 cows were sampled from the abattoirs and ovum pick up conducted on 26 cows. At slaughter 308 ovaries containing follicles were used for the study. Some ovaries were discarded on the basis of absence of follicles and presence of cysts. Three thousand, one hundred and ten $(3,110)$ follicles were counted from the ovaries and 2,368 COC's were graded into grade A and B while 476 COC's were graded into grade $\mathrm{C}$ or discards. Two hundred sixty six $(8.55 \%)$ of COC's were lost during aspiration and $1658 \mathrm{COCs}$ were taken through the IVEP process.

The process had conception rate of $46.5 \%$ and produced 17 healthy calves. Fifty seven technical staff members have been trained on the same technologies across East Africa - mainly from Kenya, Uganda and Ethiopia. 


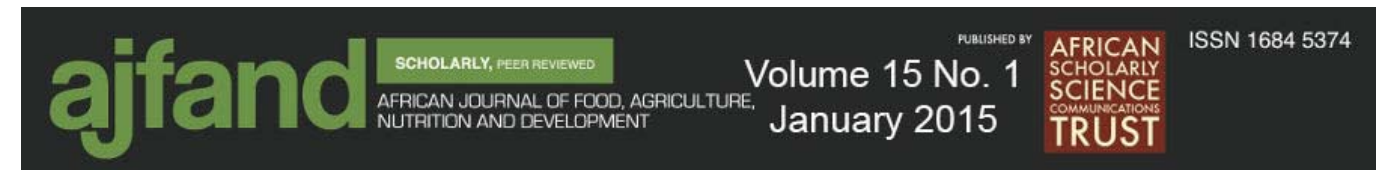

\section{DISCUSSION}

\section{Review of the Technical aspect of the Technology \\ In-vitro Embryo Production (IVEP) \\ Media and Stock Solutions}

Media and stock solutions for in vitro embryo production need to be sterile, accurately done. The use of a Laminar flow Cabinet for sterility is indispensable to avoid any contamination. Using analytical balance with readability of at least $10 \mu \mathrm{g}$ and accuracy of $\pm 0.1 \mu \mathrm{g}$ is very important. All components of the medium must be of the highest grade possible.

To achieve good standards on specifications of media and stock solutions, the following important factors need consideration.

\section{i) Water}

A Millipore-Q system or its equivalent should be used to produce high quality pyrogenfree water with a resistance of $>18$ megahms-cm. This study utilized this kind of water.

\section{ii) Glassware}

Glassware for media preparation should be designated solely for this purpose. Glassware to be used must be sterile and pass through the standard tissue culture cleaning procedure. This study used glassware that was double cleaned and sterilized through a glassware autoclaving system.

\section{iii) Disposable Materials}

Disposable supplies such as Petri dishes, acrodisc filters, centrifuge tubes for media storage and pipette tips used during IVEP make the IVEP work seem expensive but the use of disposable materials is more beneficial and convenient in terms of eliminating contamination and ensuring purity and safety of the prepared media and the produced embryos. This study used disposable materials purchased from Nutricell Company in Brazil and all were stored within clean cabinets of the laboratory. 


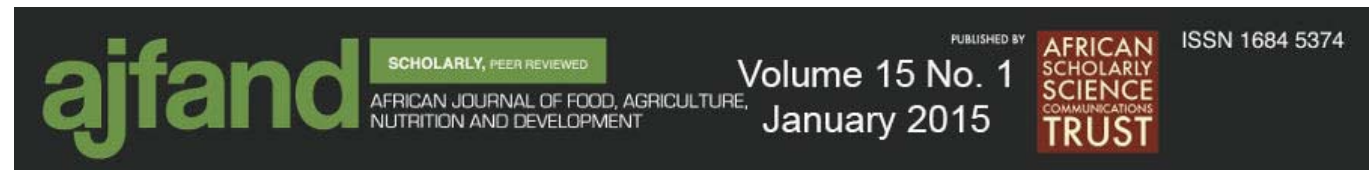

iv) Filtration

The use of a $0.22 \mu \mathrm{m}$ filter is recommended, considering that the size of the smallest bacteria is $0.3 \mu \mathrm{m}$. In this study, use of $0.22 \mu \mathrm{m}$ filter used and the first few drops were discarded to remove any contaminants from the filter and the container. The remaining volume was filtered again and stored in a refrigerator.

\section{v) Embryo Culture and Working Area}

The culture media used for the growth of oocytes and embryos is highly prone to contamination. The $95-100 \%$ humidity and $38.5^{\circ} \mathrm{C}$ temperature conditions for growth within the incubator are some of the factors that enhance the growth of any contaminating organisms present within the culture media. Sources of contamination could be air borne and/or from surfaces of the working area, or from any materials used in the preparation of the culture media. The following measures were implemented in this study to avoid contamination of the cultured oocytes and embryos.

a) Working space was separated, with various designated areas; dirty benches for aspirations, clean benches for media preparation and sterile spaces for oocytes and embryo handling and manipulations. The interior laboratory space containing the incubators was out-of-bounds for all external people, who were only allowed to view the procedures through a transparent glass wall.

b) Workers covered their hair, mouth and nose, and used disposable hoods whenever conducting oocyte and embryo culture techniques.

c) Disinfectant such as $70 \%$ alcohol was applied to the working surfaces before the preparation of the culture media and prior to any embryo production techniques.

d) Hands had to be washed first with clean water and sterilized with $70 \%$ alcohol before handling the materials for media preparation and embryo production.

\section{vi) Storage and Maintenance of Media and Reagents}

In order to improve on IVEP results, this study ensured the expiry date of all reagents and media were noted and cross-checked at all times prior to use. Stock solutions and the media were kept in their manufacturers' prescribed storage conditions. To maintain the viability and efficacy of media and reagents, preparation of well labeled aliquots was done to avoid frequent open access to the stock solutions to eliminate any possibility of contamination. Storage containers were sealed securely to avoid any moisture contamination that tends to alter the $\mathrm{pH}$ or osmolality of the media. In our system, a folder/ file with all standard preparation procedures and also with documents showing important information on preparation and expiry dates for all media and reagents was available within the laboratory.

\section{Collection of Ovaries}

Pre-slaughter record parameters were obtained prior to slaughter and or OPU to track genetics of embryos produced. Records were entered into a well-designed data collection form. Ovaries should be collected within 10 minutes after the slaughter of the animals, and kept in a sealed container containing physiological saline $(0.9 \%$ 


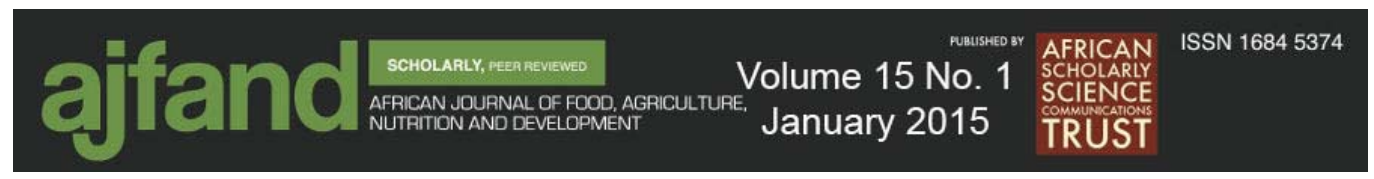

sodium chloride with either $1 \mathrm{ml} / \mathrm{L}$ Gentamycin or $100 \mu \mathrm{m} / \mathrm{ml}$ Streptomycin and 100 $\mathrm{IU} / \mathrm{ml}$ Penicillin), at a temperature ranging from $28-33^{\circ} \mathrm{C}$. Careful observation of the temperature of the saline is important to maintain the viability of the ovaries during the period of collection. In our system, the prepared saline was left to stand at $38^{\circ} \mathrm{C}$ overnight and the gentamycin was added in the morning of collection before transfer to a thermos flask. Temperature check-ups were done regularly at the slaughterhouse.

The time interval between animal slaughter and oocyte recovery from the ovaries, and the temperature of the holding medium is also important. In general, the time spent between ovary collection and oocyte recovery should be within 3-4 hours and a 30 $33^{\circ} \mathrm{C}$ temperature range of the physiological saline should be maintained. However, in cases where the slaughterhouse is far distant from the laboratory, a decrease in the temperature is recommended, although the temperature should still be kept above $28^{\circ} \mathrm{C}$ to maintain the development potential of the oocytes. Prolonging the period of ovary collection may significantly affect the viability of the oocytes for IVEP. It is recommended that oocytes should be collected from the ovaries at most within a 6-hour period. Beyond six hours, development competence of the oocytes is greatly compromised. In our system, the slaughterhouses were located $10 \mathrm{kms}$ from the laboratory and the time taken between ovary collection and delivery to the laboratory was 3-4 hrs.

\section{Oocyte Recovery}

Before oocyte recovery, it is necessary to wash the ovaries in fresh sterile physiological saline (without antibiotics) to remove any contaminants. Thereafter, briefly rinse the ovary in $70 \%$ ethyl alcohol to eliminate surface organisms before beginning the oocyte recovery procedure. The ovaries are then dried lightly with sterile paper towels and primary oocytes are recovered from 1-6 mm vesicular follicles. It was important to avoid recovery of oocytes from big follicles (beyond $6 \mathrm{~mm}$ ) because they contain secretions that tend to cause jell formation in the aspirates. In such cases the retrieval of oocytes during searching is negatively affected.

Recovery of the oocytes from the ovaries may be done by either of the following techniques:

- Oocyte aspiration using needles

- Slicing the ovaries using scalpel blade

- Follicle dissection from ovaries

- Ovum pick-up from live animals

For the ovaries collected from slaughtered animals, the aspiration method was commonly employed because of the convenience associated with it. Aspiration of oocytes was done using a needle attached to a 10-ml syringe. To avoid disruption of the surrounding cumulus cells, an 18-gauge needle was used. Possible toxicity associated with syringes containing rubber plungers and siloxane lubricants was avoided by using only sterilized syringes with plastic plungers.

One of the difficulties associated with the aspiration approach is the fact that oocytes may only be retrieved from about $60 \%$ of the punctured follicles. To minimize this 


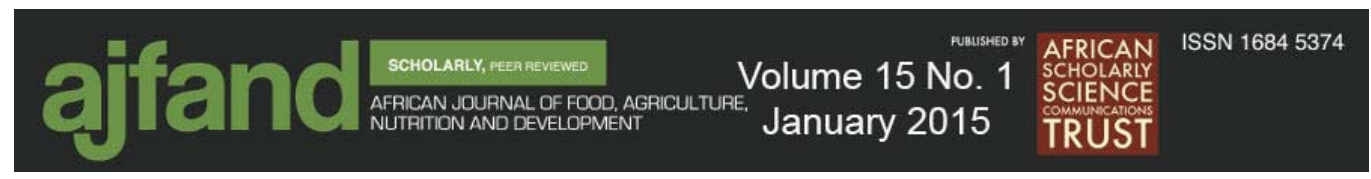

effect, it was necessary to prime the needle and syringe with approximately $0.25-0.5 \mathrm{ml}$ of aspiration medium. This is to provide the initial fluid that helps ensure the retrieved oocytes are within the fluid dispended from the syringe.

The aspiration medium used was Tyrosine-albumin-pyruvate (TALP)-HEPES (4-(2hydroxyethyl)-1-piperazineethanesulfonic acid) but also modified phosphate-buffered saline was used occasionally, especially, during training sessions. Aspiration medium can be prepared once, stored in a refrigerator and made available for one week's activity. However, it is very important to pre-warm the aspiration medium up to $38^{\circ} \mathrm{C}$ before use.

After aspirations are made from follicles of each ovary, the contents in the aspirating syringe are slowly transferred into a sterile centrifuge tube that is pre-moistened with TALP-HEPES and the oocytes are allowed to settle at the bottom of the tube that is placed into a rack within a warm water bath set at $38^{\circ} \mathrm{C}$. The transfer of fluids containing the oocytes into the tube is done slowly with minimal disruption of the cumulus-oocyte complex (COC). When this is done from the last ovary of the day, the precipitate containing the oocytes that had settled at the bottom of the tube are selectively picked using a sterilized Pasteur pipette and transferred into sterile Petri dishes containing TALP-HEPES for subsequent searching, grading and counting of recovered COCs. The recovery work of COCs should be done in a sterile environment within room temperature of $25^{\circ} \mathrm{C}$.

Sometimes slicing of the ovaries is done in order to enhance retrieval of numbers of COCs. However, the method is more tedious and requires longer period of retrieval and is thus not recommended whenever manipulating a large number of ovaries because the extended period of oocyte retrieval would negatively affect oocyte competence. In this case, the method was used only when few ovaries were collected.

Follicle dissection is also a method that can be used. This method is effective in retrieving good-quality oocytes as reviewed extensively by Gordon [10]. However, it also increases the time needed for the retrieval process. In this study, this was only used during research.

Ovum pick up can also be done from live cows using ultrasound guided follicular aspiration through a needle passed trans-rectally as shown demonstrated in Fig 2. 

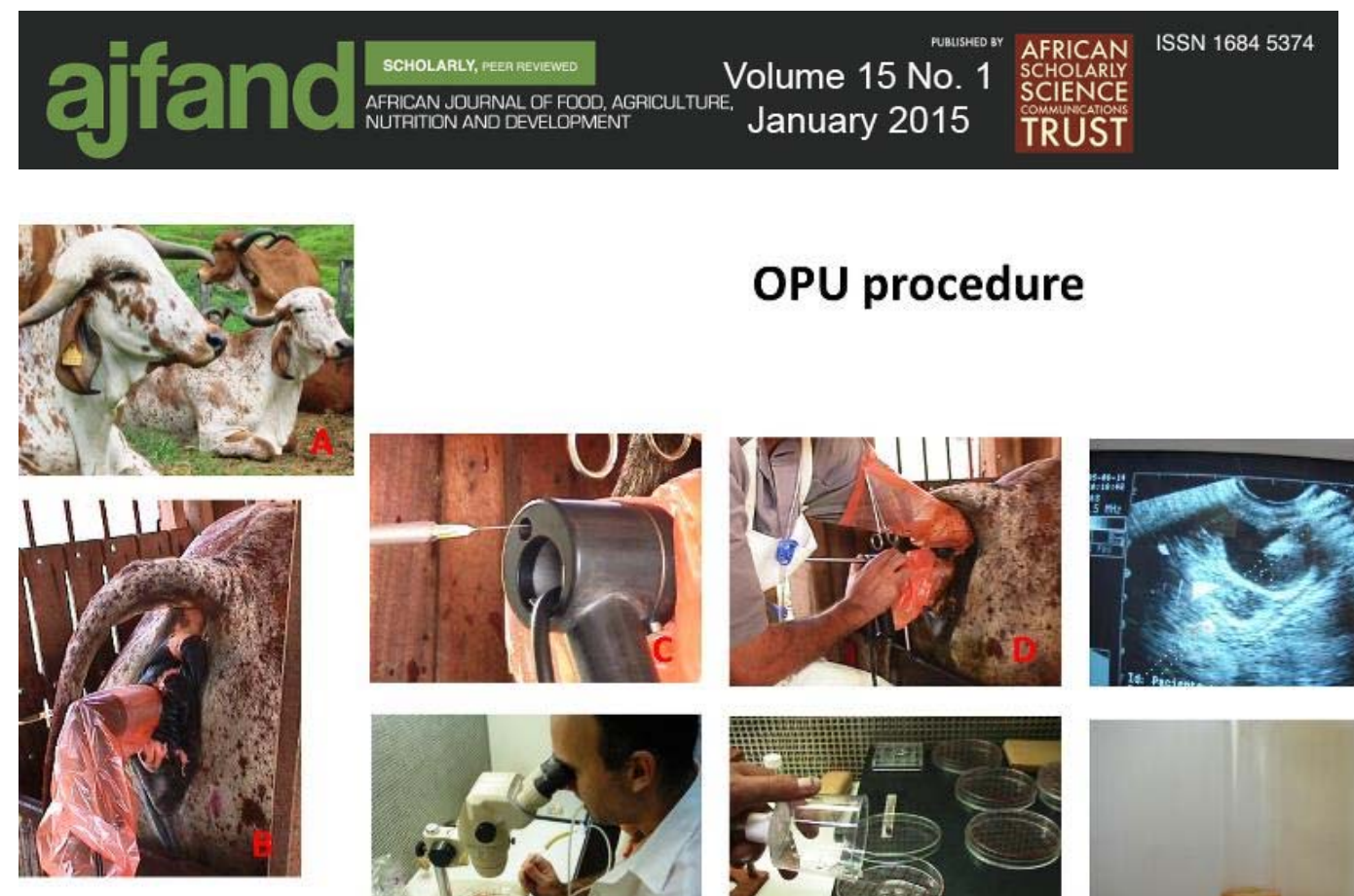

\section{OPU procedure}


Figure 2: Photographic demonstration of the OPU procedure; donor cows selected $(A)$, then ultrasound device inserted into the vagina $(B)$, needle put into the device $(\mathrm{C})$, the expert picks the ovary towards the intravaginal ultrasound device $(D)$, the follicles are viewed through the ultrasound monitor $(E)$, the follicles are aspirated into a tube $(F)$, the follicles are poured into a petri dish (G), the follicles are searched $(\mathrm{H})$, the follicles are recovered into another petri-dish (I), grade A COC (J), grade A COCs are loaded into thermos flask (K), the COCs are delivered to the laboratory $(\mathrm{L})$

This technique is used for repeated collection of COCs from top grade cows. The method was also used within the IVEP system reported here.

\section{Selection of Oocytes for in Vitro Maturation}

Once the COCSs are located within the Petri dish during searching under a microscope at X10 objective, the COCs were picked using micropipettes with a bore diameter of about $200 \mu \mathrm{m}$ and transferred into a dish of fresh pre-warmed TALP-Hepes washing medium. The plastic tip used in picking the COCs must have a bore diameter wide enough to avoid disruption of the cumulus cells surrounding the oocytes. The presence of cumulus cells surrounding the immature oocytes is a prerequisite both for successful maturation of the oocyte and for embryo competence. 




Only oocytes with compact multilayered cumulus investment and evenly granulated cytoplasm should be selected for in-vitro maturation. It should be noted, however, that the aspirated COCs may have a differences in their appearances [11]. Some will be denuded (with no cumulus cells attached to the zona pelucida), others being partly denuded (with one sided swollen cumulus cells, and others with an already expanded or spider-like cumulus cells. Others will also have uneven cytoplasm. Not all oocytes should be used for IVEP because they lack the potential to undergo normal maturation and will eventually end up as degenerated COCs after in-vitro fertilization. Further details on IVEP of Boran cows were described by Muasa in 2010, which contains a chapter providing guidelines on COC grading for IVEP [11].

\section{In Vitro Maturation of COCs}

Prior to transfer to an in vitro maturation (IVM) medium, selected COCs must be washed 2-4 times in fresh pre-warmed TALP-Hepes medium and finally washed in invitro maturation medium. The medium used for in-vitro maturation (IVM) of oocytes varies among laboratories. It should be considered that the culture medium employed in IVM may affect the proportion of oocytes that reach metaphase II and become capable of undergoing fertilization. It also influences subsequent embryo development [12]. The culture media employed in maturation of oocytes can be broadly divided into simple and complex.

Simple media are usually bicarbonate-buffered containing basic physiological saline with pyruvate, lactate and glucose. The main difference between various types of simple media is in their ion concentration and energy sources. The media are generally supplemented with serum or albumin, with trace amounts of antibiotics added (penicillin, streptomycin, gentamicin).

Complex media, on the other hand, contain, in addition to the components of the simple media, amino acids, vitamins, purines and other substances, mainly at levels they are found in serum. Fixed nitrogen is present as free amino acids.

This study used a widely-used complex media for IVM of cattle oocytes: Tissue Culture Medium 199 (TAC-199) with Earle's salt, L-Glutamine and $25 \mathrm{mM}$ HEPES supplemented with $10 \%$ heat inactivated fetal calf serum (this can also be calf serum, steer serum or serum from cow in estrus), and supplemented with $1 \mathrm{ug} / \mathrm{ml}$ of follicle stimulating hormone (FSH), Luteinizing Hormone (LH) and estradiol.

Other IVM media can be used such as Synthetic Bovine Oviductal Fluid medium and others. All others, however, require further supplements in order to promote maturation.

During IVM, extensive redistribution of intracellular organelles occurs. In addition to extensive cumulus expansion that occurs during maturation of oocytes during IVM, the mitochondria migrate to occupy a perinuclear location, and the cortical granules migrate outward to lie just beneath the vitelline membrane of the oocyte. At all times, the critical requirement of successful oocyte maturation is the capability to undergo normal embryonic development after sperm penetration and fertilization. An extensive description on how to evaluate and detect matured oocytes after IVM has been 


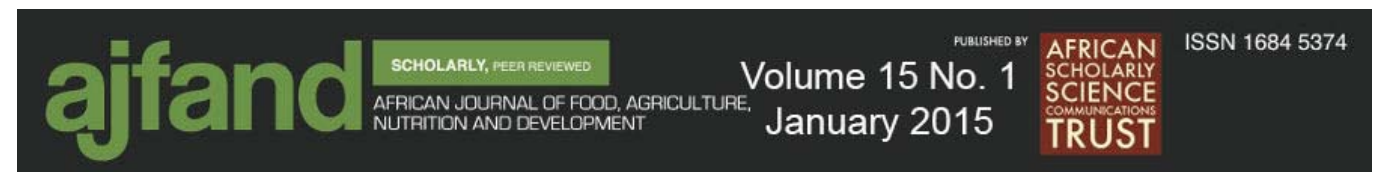

described in depth by Muasa [11]. However, the following are the important considerations one must take into account during IVM procedure:

\section{a) Water quality}

Water is the major constituent of any IVM medium and due to the presence of several basic contaminants such as ionized and non-ionized solids and gases, particulate matter, microbials and pyrogens, the use of ultrapure water is highly recommended.

\section{b) Buffering Systems and Osmolality}

The $\mathrm{pH}$ of the IVM media changes drastically whenever exposed to non- $\mathrm{CO}_{2}$ controlled atmosphere. Hepes or phosphate-buffered media for short-term work with oocytes and embryos does not require a carbon-dioxide controlled gas phase to maintain a relatively constant $\mathrm{pH}$. For this reason, these media should be commonly used for washing, and for storage of oocytes and embryos outside the incubator. The optimum range of osmolality of the IVM medium should range between 275 and $285 \mathrm{mOsmol}$. If the osmolality of the IVM medium is not within this range, it should be discarded and a new one prepared. In some instances, the measured osmolality of a medium is less than the calculated value. This can be attributed to the incomplete dissociation of ions once they reach $\mathrm{mM}$ concentrations. In our system we utilized TALP-Hepes as washing media and osmolality of IVM made was $280 \mathrm{mOsmol}$.

\section{c) Flux Culture}

This involves the regular, gentle agitation of the culture dishes to prevent the attachment and subsequent differentiation of cumulus cells within maturation tubes. Micro droplets of IVM media covered by oil offer advantages including preventing or reducing the evaporation of water, protection from microbial contamination, attenuation of temperature and gas fluctuations, and ease of examination during culture period. This practice is advisable.

\section{d) Maturation Time}

Some discrepancies exist in the length of time needed for IVM of cattle oocytes. The culture medium and the supplements used, as well as the quality of oocytes, influence the length of time required before oocytes attain the maturation stage (Metaphase II). This study showed that while there was no significant difference between maturation period in terms of maturation and cleavage rates, there was a significant difference in final blastocyst yield in favor of the $24 \mathrm{~h}$ maturation period. Thus, the $24 \mathrm{hr}$ maturation period for IVM of oocytes is recommended.

\section{e) Other considerations}

\section{i) Antibiotic cover}

This provides cover against growth and proliferation of microorganisms during the period of IVM culture. The concentration of antibiotics must be non-toxic and their inclusion in the medium needs to be combined with rigorous standards of hygiene in the IVM laboratory. 




ii) Light environment

As part of routine maturation, fertilization and culture, the cattle embryos are inevitably exposed to varying amounts of light. The lesions caused by prolonged exposure of high light intensity under the microscope may contribute to embryo development failure. In principle, oocytes and embryos should not be over exposed to light longer than is necessary. Maturation and other events normally occur under conditions of darkness in the animal's reproductive organ and in our case, the laboratory windows were covered with opaque curtains.

iii) Temperature and Gas phase

The success of IVEP is temperature-dependent. Changes in temperature exposure to oocytes can lead to temperature shocks that can induce chromosomal abnormalities. The temperature range of $38-39^{\circ} \mathrm{C}$ is found to be ideal for cattle oocytes during IVEP. The incubation temperature was set at $38.5^{\circ} \mathrm{C}$ in all IVEP procedures. Gas phase was also observed to have a very big influence on the success of Boran IVEP. Development to the morula and blastocyst stage of the embryo was found to be highly dependent on the gaseous environment of the incubator during IVEP. The best is an environment of $5 \% \mathrm{CO}_{2}, 5 \% \mathrm{O}_{2}$ and $90-100 \%$ humidity.

\section{iv) Culture supplements}

The supplements usually added to the culture medium are the following:

v) Proteins

The most commonly used protein source is serum added in a concentration of 5 to $10 \%$. Proteins in serum have macromolecules attached such as hormones, vitamins and fatty acids, as well as chelated metal ions and pyrogenes. Bovine serum albumin was used for protein supplement. The role of protein in the culture media may not only be a fixed nitrogen source, but also as a chelator of toxic metal ion. In this system we used $10 \%$ fetal calf serum.

\section{vi) Hormones and Growth factors}

Some of the hormones used as supplements include FSH, LH, oestradiol and prolactin. The direct action of these hormones on oocyte maturation and early development of embryo are not well established, but their addition to the culture media improves development of pre-implantation stage embryos. Growth factors added include IGF (insulin-like growth factor) and EGF (epidermal growth factor). These growth factors have mitogenic effects and also stimulate RNA and protein synthesis. In our? case we only used $1 \mu \mathrm{g} / \mathrm{ml}$ of FSH, LH and estradiol.

\section{vii) Culture Apparatus}

In thsese experiments, it was observed that the type of culture apparatus used for in vitro culture of oocytes and embryos affected the outputs of in vitro maturation and also development of pre-implantation stages of the embryos. The use of ordinary Petri dishes was not as beneficial as the use of the Nunc 500um deep holed petri dishes, which was found to greatly enhance embryo development. 


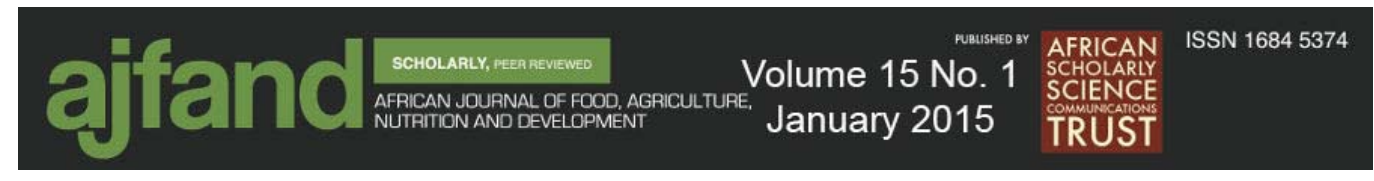

\section{Sperm Treatment and Capacitation}

To ensure a successful fertilization of the oocytes during IVEP, the sperm cells that are used must be viable, motile, capacitated and capable of expressing acrosome reaction. Thus, the cells must bind to the zona pellucida of the oocyte and pass through its vitelline membrane. This is only possible if the cells are able to fuse with the oolemma and be incorporated into the oocyte. This ability is normally acquired by the sperm cells as they pass through the epididymis through maturation process and within the female genitalia through the process of capacitation.

The process of capacitation is critical for fertilization. Only capacitated cells can undergo acrosomal reaction, a vital step that aids the cell to penetrate the ova to achieve fertilization. Capacitation is a process that involves complex biochemical and physiological changes within the sperm cell. During this process, gradual removal or alteration of the acrosomal protective coat from the sperm surface occurs. This then permits exposure of acrosomal enzymes needed for effective penetration of the ova and also exposure of sperm cell receptors that aid in binding onto the zona pellucida receptor sites.

In the laboratory, capacitation procedures are aimed at stimulating the sequence of events that normally occur in the female reproductive tract. The seminal proteins and any dead cells within the ejaculated and/ frozen semen are removed thorough either a process of swim-up through capacitation medium or by centrifuge process through elevated ionic strength. The following are the components of an effective capacitation medium.

\section{a) Bovine Serum Albumin (BSA)}

Bovine serum albumin (BSA) plays a key role in removing cholesterol and/or zinc ion from the sperm cell. Bovine serum albumin protein has considerable binding capacity for both cholesterol and zinc molecules. Cholesterol and zinc usually stabilize sperm cell membrane and must be removed during capacitation to enable acrosomal reaction and penetration of the ova.

\section{b) Heparin}

Heparin is a glucoseaminoglycan molecule added to the medium to mimic in-vivo heparin-binding proteins present usually present in female genitalia during capacitation. Heparin proteins play a role in fertilization by inducing the process of capacitation.

\section{c) Caffeine}

Caffeine is a cyclic nucleotide phosphodiesterase inhibitor used as a motilitystimulating agent in bull sperm. When caffeine inhibits phospodiesterase within the sperm cell the intracellular accumulation of cyclic adenosine monophosphate (cAMP) occurs resulting into activated sperm motility.

d) Calcium Ionophore

Calcium is added to increase calcium ion $\left(\mathrm{Ca}^{2+}\right)$ content of the medium to facilitate sperm motility and ova penetration during fertilization. 


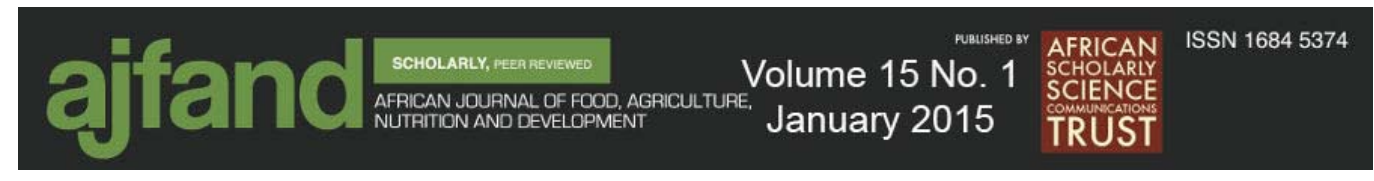

\section{Swim-up technique of sperm capacitation}

In-vivo sperm capacitation usually takes about $6 \mathrm{~h}$ within the female reproductive system. In the laboratory, we conducted a swim-up technique for this process.

Prior to swim up, the semen is evaluated by placing a drop on a pre-warmed glass slide and checked under a microscope for vigour and motility. Only semen that attained over $50 \%$ motility was used. For swim-up, $0.25 \mathrm{ml}$ of the semen was then pipetted and gently released at an angle of $45^{\circ}$ at the bottom of the $10 \mathrm{ml}$ sterile centrifuge tube containing $1.0 \mathrm{ml}$ of swim up -TALP medium as shown in figure 2 above.

The swim up was done in a $5 \% \mathrm{CO}_{2}$ buffered incubator for 60 minutes at $38.5^{\circ} \mathrm{C}$ to allow the motile sperm to swim up through the medium as they also at the same time undergo capacitation [13]. After one hour, about $0.8 \mathrm{ml}$ of the upper layer of the medium (supernatant) with the vital sperm, was pipetted into a $15 \mathrm{ml}$-apirogenic tube and centrifuged at 10,000 rotations per minute (RPM) for 5 minutes at room temperature. The supernatant was then discarded and the sperm cell pellet located at the bottom of the tube was re-suspended with 100ul of fertilization (Fert-TALP) medium.

\section{In Vitro Fertilization (IVF)}

Successful IVF can only take place with appropriate preparation of sperm and oocyte, and also in culturing conditions that are favorable to the metabolic activity of the male and female gametes. In cattle, IVF of in vitro matured oocytes is usually carried out 1724 hours within an incubator set at $38.5^{\circ} \mathrm{C}$, at $95-100 \%$ humidity and $5 \% \mathrm{CO}_{2}$ [14]. In vitro fertilization (IVF) is carried out in a $100 \mu \mathrm{l}$ micro droplets of IVF medium containing sperm cells at $1 \times 10^{6} \mathrm{sperm} / \mathrm{ml}$ concentrations, to fertilize $10-15$ oocytes. In order to avoid going into the laboratory at odd hours, it is recommended, as we practiced, to conduct IVF for $24 \mathrm{hrs}$ [15]. The oocytes were processed by washing twice in pre-warmed IVF medium.

\section{Sperm preparation for IVF}

The right concentration of the sperm was determined by use of a Neubauer chamber using a 1:20 dilution factor as previously described $[16,17,18]$. Five micro litre of the capacitated semen after swim up was mixed with $95 \mathrm{ul}$ of water in order to kill the sperm and also achieve a 1:20 dilution factor. Then this was mixed thoroughly and loaded into both sides of Neubauer chamber and the numbers of sperm cells in both fields of the Neubauer chambers counted under a microscope. The total number of sperm cells counted was used to determine the spermatozoa concentration (sperm cells $/ \mathrm{ml}$ ). Experience indicated that whenever using a dilution factor of 1:20 in Neubauer chamber to calculate sperm concentration for IVF, a final concentration of $1 \times 10^{6} \mathrm{sperm} / \mathrm{ml}$ can be obtained by using semen volume equivalent to the volume of IVF droplet divided by the number of counted cells after taking into account the percentage of the post-thaw motility $[16,17]$. Thus Volume of semen to use $=$ volume of IVF droplet / (no. of counted cells $/ 10 \mathrm{x} \%$ post thaw semen motility). 


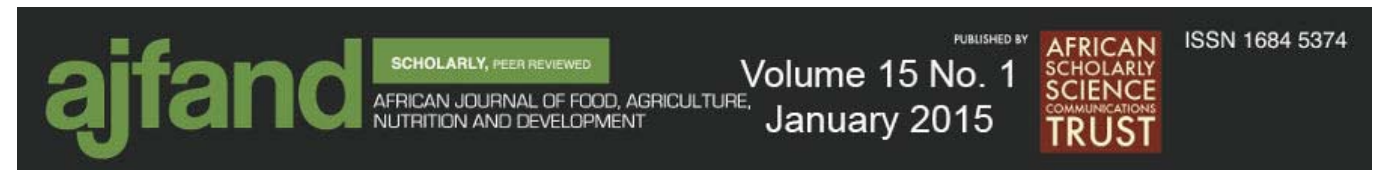

The calculated final volume of semen was then drawn and added to a droplet of IVF to be part of the 100ul of the final IVF droplet. Then the oocytes are added in the subsequent volume of IVF remaining to make up the required final 100ul IVF droplet.

\section{In-Vitro Culture (IVC) of Embryos}

In this system the zygotes were co-cultured with a monolayer of their cumulus cells. The presence of a somatic cell monolayer in the culture medium during in-vitro culture of the developing embryo enhances its developmental potential. The layer supports and provides the developing zygotes with a comfortable environment by secreting some growth factors to enhance their development. It is important to use culture medium that has been pre-conditioned in the incubator at least 1-2hrs prior to in vitro culture (IVC).

In vitro culture (IVC) is usually carried out for seven days. At the third day, in order to remove any metabolic waste resultant during cumulus-oocyte complex maturation and provide the embryos with a fresh medium that could sustain their metabolic requirements, half of the medium is drawn out and another fresh one added $[17,18,19]$.

Before IVC, oocytes are washed 2-4 times in a washing medium (culture medium) to remove the excess sperm cells attached to the zona pellucida. It is important to remove excess sperm cells to avoid the presence of dead and denaturing cells that may contaminate the IVC medium [20]. In vitro embryo culture, like other procedures of IVEP is carried out inside a water-jacketed incubator with $5 \% \mathrm{CO}_{2}$ at $38.5^{\circ} \mathrm{C}$.

It is advisable to check for cleavage rate three days ( $72 \mathrm{hrs})$ after the initiation of IVC whenever changing the IVC medium. At that time, most of the zygotes will be over 5cell stage. Blastocysts used during embryo transfer are expected to be formed at day seven of IVC. During the evaluation times, gentle shaking of the culture dish is advised to allow for a uniform environment among embryos, and to allow for unified distribution of any autocrine growth factors that the embryo may have secreted. Also recommend that oocytes that have not cleaved are removed from the culture droplets at those times.

\section{Other Important Considerations for Boran IVEP}

\section{a) Cleaning of Glassware}

Cleanliness of the glassware is of utmost importance. Used glassware is rinsed thoroughly with tap water and soaked in detergent overnight. The following day, glassware is rinsed thoroughly at least 20 - 40 times in tap water, and then submerged in a 3-5\% hydrochloric acid $(\mathrm{HCl})$ for a minimum of 2 hours. It is then rinsed in running water for about 15 minutes, and rinsed again 20 - 40 times in tap water. Thereafter, glassware is rinsed with distilled water and placed upside-down to dry by heating it at $120^{\circ} \mathrm{C}$ for $2-4$ hours. Glassware is then packed separately in aluminum foil and sterilized by heating at $120^{\circ} \mathrm{C}$ for 4 hours.

An ultrasonic cleaner is also used for heavily soiled and difficult to wash glassware such as volumetric flash, test tube and volumetric pipettes. Cleaning by sonication takes around 15 minutes in hot water. After sonication, glassware is rinsed 20 - 40 times with 


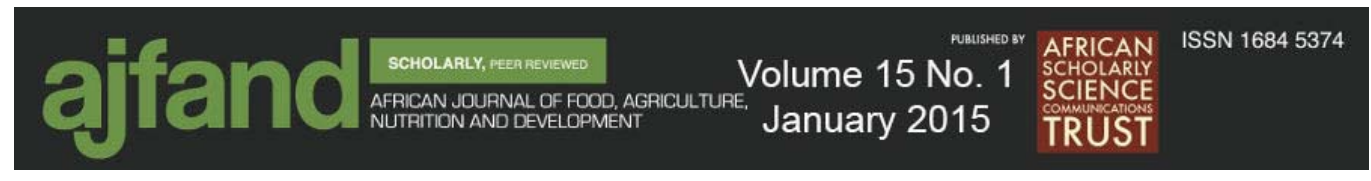

tap water and finally rinsed with distilled water. Thereafter, the drying and sterilization protocol described above is practiced.

\section{b) Availability of Back-up Equipment and Equipment Repair Kit}

In some cases, equipment needs repair when it malfunctions or becomes defective. Back-up equipment must be available, to avoid compromising the IVEP activity. Staff working at the laboratory must be familiar with any repair measures that may need to be implemented. Prompt repairs are a requirement. It is important to have spare gas cylinders $\left(\mathrm{CO}_{2}\right)$, back-up power sources and a spare incubator.

\section{Discussion of the Basic Data}

An average BCS of 2.0 for the slaughtered Boran cows is reported in this study. Some authors recommend that ovaries be collected from donor cows of superior body condition score that exceeds 2.0 [10]. This study also demonstrates that Boran cows with a body condition of $1.5-4.5$ were able to produce competent COC's and viable embryos. However, in order to produce optimal results in IVEP for the Boran breed, further studies may be necessary to shed more light on influence of nutrients and BCS of the cow on its embryo production in vitro.

\section{Estrous synchronization}

Estrous synchronization is an old biotechnology that has been used widely in Kenya. The technology ensures that all cows treated together come on heat around the same time. This process can be used to control follicular development during ovum pick-up sessions and time heat occurrences so as to allow for timed embryo transfer (TET). The study utilized the technology involving use of hormones to knock-off corpus luteum (CL) from cycling animals at the same time so as to initiate similar patterns of follicular development in all treated animals.

Given that hormonal control of the estrous cycle eases reproductive management and improves efficiency of cattle operations, the plan was to provide practical applications of the manipulations of estrous cycle of Boran cows so as to plan ovum pick-up sessions from donor cows and/or achieve mass embryo transfers to recipient cows.

\section{Ovum Pick UP (OPU) in Live Animals}

Ovum pick up is a technology used to harvest ova from live animals. This technique requires a special ultrasound scanning machine that is used to retrieve good numbers of quality ova from a donor cow per harvesting session. The plan is to utilize this technology and harvest ova from farmers keeping cows of known top genetics.

It is known that in cows two to four waves of follicles (source of ova) develop in a given estrous cycle. Every wave has recruitment, selection and dominant phases. When ova harvesting is done either during recruitment or selection phase, over 70 ova per ovary per cow can be obtained.

\section{Protocol used for Field Estrus synchronization to knock-off $\mathbf{C L}$}

Prostaglandin (PGF) is a naturally occurring hormone. During the normal estrous cycle of a non-pregnant animal, PGF is released from the uterus 16 to 18 days after the cow 


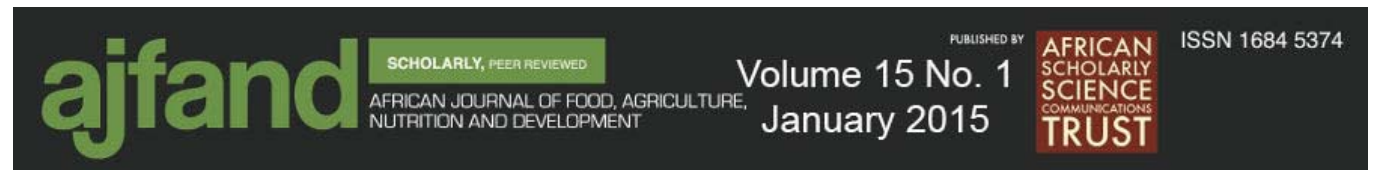

was in heat. This release of PGF functions to destroy the corpus luteum (CL). The CL is a structure in the ovary that produces progesterone and prevents the animal from returning to estrus. The release of PGF from the uterus is the triggering mechanism that results in the animal returning to estrus every 21 days. Commercially available PGF (Lutalyse, Estrumate, Prostamate) can be used to knock-off the CL from any cycling cow at a predetermined time convenient for heat detection and breeding.

The major limitation of PGF is that it is not effective on animals that do not possess a CL. This includes animals within 6 to 7 days of a previous heat, prepubertal heifers and postpartum anestrous cows. Despite these limitations, prostaglandins are the simplest method to synchronize estrus in cows. In our system we used $500 \mu \mathrm{g}$ cloprostenol $(2 \mathrm{ml}$ of Lutalyse) injected intramuscularly. Heat detection was observed keenly after $48 \mathrm{hrs}$ twice a day (7-730 $\mathrm{h}$ and 18-1830 h) when animals were in the farm house. Also, heat detection was also done throughout the day as the animals grazed in the fields.

\section{Embryo Transfer}

Embryo transfer is a technology of placing a seven day old embryo in the uterus of a recipient cow. The transfer embryos can be flushed from the uterus of a donor cow or be obtained from an in-vitro embryo production (IVEP) laboratory. Obtaining embryos from donor cows requires that such animals are treated beforehand to enable superovulation to occur in them. In addition, this process requires that recipient cows are also hormonally treated to achieve uterine synchrony with the donors for effective transfers. By contrast, hormonal treatment steps are eliminated whenever embryos are produced in an IVEP setup, making this method of obtaining embryos a better option. Thus, as an alternative source of embryos to the already existing conventional embryo transfer in Kenya [4], IVEP is technically feasible and can allow for a predictable supply of embryos from ovaries of slaughtered or live selected cows.

Embryo transfer is carried out after the cow is prepared by giving it 3-5 $\mathrm{ml}$ of lignocaine epidural anaesthetic at the sacro-coccigeal space to allow for easy rectal manipulations. Then the perineum is washed with water, and disinfected. The embryos were then loaded into the embryo transfer (ET) gun as the embryo transfer expert examines the ovaries of the cow so as to ascertain into which uterine horn to transfer the embryo.

\section{CONCLUSION}

Utilization of the IVEP technology in Kenya can be used to carry out value addition to indigenous cows. There can be situations where farmers keeping low-grade cows can enter into commercial contracts to provide these cows as surrogate recipients for production of heifer calves. In the process, owners of such cows will have a valued product to sell for income earnings and be left with milk for use by family members. This avenue can be used to raise household revenues and attract many more farmers to engage in farming, leading to improved national economic and food security.

When IVEP and OPU technologies are combined, OPU enables a cow to provide over 100 ova per ovary per month for sale. This innovation can be applied to exploit this 




special potential of the cow to equip owners of such cows with an opportunity to obtain and sell 100-150 ova/cow/month.

Countries such as Brazil have embraced innovations coming out of IVEP and OPU to turn around the livestock sector recent years. In the applied context, the farmers, the private industry and the embryo transfer practitioners in Brazil entered into publicprivate partnership on mutual benefit relationships and revolutionized the dairy sector [22]. In the same way, the authors hope to see this technology widely utilized in Kenya, due to its massive potential benefits to the dairy sector. It is noted that although ovarian follicular dynamics has been documented in some Zebu cows, literature on the same for the Boran cow is scarce. Thus, further studies are recommended on ovarian follicular dynamics of the Boran cow so as to provide this information in order to rapidly optimize capacity of this breed through utilization of in vitro embryo production and ovum pick reproductive technologies. Also cost-benefit analysis studies are recommended before the establishment of this technology in Kenya. The authors recommend that IVEP is a process that requires optimization of any described protocols to suit the methodology and materials involved in any set ups. Thus caution should be taken wherever adopting protocols developed elsewhere in another laboratory.

\section{ACKNOWLEDGEMENT}

The authors acknowledge Heifer Project International for funding this work and International Livestock Research Institute (ILRI) for providing space for laboratory facilities. Authors also appreciate University of Nairobi for providing time for the technical experts and recipient cows and Kapiti Plains for providing donor cows. Abattoirs that allowed harvesting of ovaries are also appreciated. All support staff are appreciated for heat detections and herding the animals. 


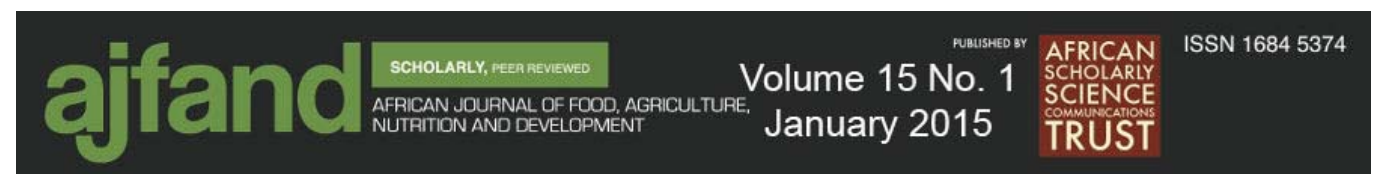

Table 1: Summary of data from In-Vitro Embryo Production process*

\begin{tabular}{|c|c|c|c|c|c|}
\hline $\begin{array}{l}\text { Follicle Size } \\
\text { group }\end{array}$ & Grade & Aspirated & Matured & Cleaved & Blastocyst \\
\hline $1-3 \mathrm{~mm}$ & A & 393 & 368 & 180 & 21 \\
\hline $1-3 \mathrm{~mm}$ & $\mathrm{~B}$ & 394 & 341 & 160 & 38 \\
\hline $1-3 \mathrm{~mm}$ & Discards** & 151 & - & - & - \\
\hline$>3-6 \mathrm{~mm}$ & A & 226 & 213 & 121 & 36 \\
\hline$>3-6 \mathrm{~mm}$ & B & 196 & 167 & 120 & 42 \\
\hline$>3-6 \mathrm{~mm}$ & Discards** & 97 & - & - & - \\
\hline$>6 \mathrm{~mm}$ & A & 85 & 81 & 71 & 24 \\
\hline$>6 \mathrm{~mm}$ & B & 61 & 56 & 44 & 7 \\
\hline$>6 \mathrm{~mm}$ & Discards** & 25 & - & - & - \\
\hline
\end{tabular}

Table 2: Distribution of follicles obtained from the various follicle group sizes

\begin{tabular}{ccc}
\hline Follicle Size & Number of follicles & Percent\% \\
\hline $1-3 \mathrm{~mm}$ & 1132 & 58.7 \\
$>3-6 \mathrm{~mm}$ & 575 & 29.8 \\
$>6 \mathrm{~mm}$ & 222 & 11.5 \\
& & \\
\hline
\end{tabular}




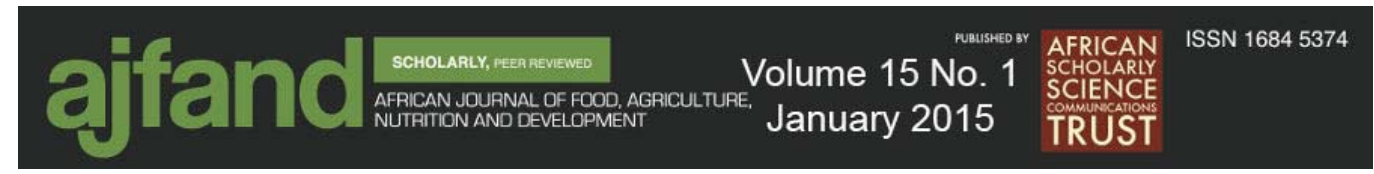

\section{REFERENCES}

1. Thornton PK, Jones PG, Owiyo T, Kruska RL, Herrero P, Kristjanson A, Notenbaert $\mathbf{H}$, Bekele $\mathbf{N}$ and A Omolo Report to the Biotechnology Department for International Livestock Research Institute (ILRI), 2006.

2. Seré C, van de Zijpp A, Persley G and E Rege Animal Genetic Resources, Information Bulletin 2008; 42: 3-27.

3. Cunningham EP and O Syrstad Crossbreeding Bos indicus and Bos taurus for milk production in the tropics. FAO Animal Production and Health Paper 1987, 68. Food and Agriculture Organization of the United Nations, Rome.

4. King JM, Pasons DJ, Turnpenny JR, Nyangaga J, Bakari P and CM Wathes Animal Science 2006; 82(5): 705-176.

5. Kahi AK, Nitter G, Thorpe $\mathbf{W}$ and CF Gall Cattle breeding in the tropics. Liv. Prod. Sci. 2000; 63:53-63.

6. McClintock SE, Ouma R, Baltenweck I, Okeyo AM, McClintock AE and E Rege Proceedings of Association for the Advancement of Animal Breeding and Genetics 2007, Australia.

7. Wildt DT Potential applications of IVF technology for species conservation. In: Fertilization in Mammals, B.D. Bavister, E. Roldan, and J. Cummins, (Eds.). Serono Symposium, U.S.A., 1990: 344-364.

8. Mutembei HM, Muasa BS Origa R, Jimbo S, Ojango JK, Tsuma VT, Mutiga ER and AM Okeyo in-vitro Fertilization in cattle and its potential practical application in Kenya. Proced 6th FVM Biennial Scientific Conference, Sept $17^{\text {th }}-19^{\text {th }} 2008,15$.

9. Mutembei HM, Muasa BS Origa R, Jimbo S, Ojango JK, Tsuma VT, Mutiga ER and AM Okeyo Delivery of appropriate cattle genotypes to Eastern African smallholder farmers through in-vitro embryo production technologiesthe technical procedures, prospects and challenges. Aspects of African Biodiversity. Proceedings of the Pan Africa Chemistry Network Biodiversity Conference, RSC Publishing 321, 2009: 84-90, ISBN: 978-1-84755-948-7.

10. Gordon I Laboratory Production of Cattle Embryos. CAB International, Wallingford, Oxon OX10 8DE, UK. 672 1994; ISBN 0851989284.

11. Muasa BS Effects of follicle size and cumulus oocyte complex grade on in vitro embryo developmental competence for Boran cows. Unpub. M.Sc. Thesis, University of Nairobi, Kenya 2010: 74-89. 




12. Aman RR and JE Parks Effects of cooling and rewarming on the meiotic spindle and chromosome of in vitro matured bovine oocytes. Biol. Reprod. 1994; 50: 103-110.

13. Parrish JJ, Susko-Parrish J, Leibfried-Rutledge ML, Critser ES, Evestone WH and NF First Bovine in vitro fertilization with frozen-thawed semen. Theriogenology'; 1986; 25:591-600.

14. Bracket BG and G Oliphant Capacitation of rabbit spermatozoa in vitro. Biol. Reprod. 1975; 12: 260-274.

15. Chuangsoongneon $\mathbf{U}$ and $\mathbf{M}$ Kamonpatana Oocyte maturation, in vitro fertilization and culture system for developing preimplantation swamp buffalo embryos using frozen thawed semen. Buffalo Journal 1991; 2: 189-198.

16. Rogers BJ Factors affecting mammals' in vitro fertilization. In: Bioregulators of reproduction. Academic Press, New York, 1981: 459-486.

17. Camargo LSA, Sá WFD, Ferreira ADM, Viana JHM and MCCD Araújo Effect of sperm concentration and oocyte-spermatozoa incubation period on in vitro fertilization of Gyr breed. Pesquisa Agropecuária Brasileira, Brasília, 2002; 37 (5): 709-715.

18. Camargo LSA, Viana JHM, Sá WF, Ferreira AM, Ramos AA and VR Vale Filho Factors influencing in vitro embryo production. Animal Reproduction 2006; 3 (1): 19-28.

19. Duran DH Studies related to the effects of Dimethyl Sulphoxide (DMSO) on the culture systems of bovine oocytes in vitro. Unpub. M.Sc. Thesis, Miyazaki University, Japan. 1996; 164: 76-81.

20. Duran DH Tsuzuki Y Ashizawa K and N Fujihara Enhanced bovine embryo development from cultured cells and conditioned medium treated with Dimethyl Sulfoxide (DMSO). In: Congress Programme of the $13^{\text {th }}$ International Congress on Animal Reproduction (Darling Harbour, Sydney, Australia) June 30 - July 4, 1996; 3: 22-18.

21. Gardner DK and M Lane Embryo Culture Systems. In: Handbook of In Vitro Fertilization, A. Trounson and D.K. Lane, (Eds.). CRC Press Inc., 1993: 85114.

22. Viana JHM and LSA Camargo 2007. Bovine embryo production in Brazil: a new scenario. Acta Sci. Vet. 35, 920-924. 